\title{
APPENDIX
}

\section{Might a Vegan Diet Be Healthy, or Even Healthier?}

\section{Introduction}

Most great apes consume a wide variety of plant foods (Nestle 1999, 214; Milton 1999). The Western lowland gorillas who live in the Central African Republic, for example, have been observed to eat over 200 different plants and more than 100 varieties of fruit (Popovich et al. 1997). Many of these plants foods are low in calories, so that the great apes must eat large quantities of them.

The human ape is an exception. With the emergence of Homo erectus about 1.8 million years ago, a transition took place towards diets that were nutritionally dense, which facilitated a significant expansion in brain size (Leonard 2014). Another factor that facilitated a further increase in brain size was the introduction of cooking about 250,000 years ago. When they started cooking, human beings benefited not only from easier mastication, but also from a greater digestibility of, and an increase in energy derived from, food (Carmody and Wrangham 2009). Whereas cooked foods did not only include animal products, it is thought that our gathering and hunting ancestors may have obtained more than half of their daily energy from animal foods (Cordain et al. 2000; Mann 2000). As animal foods provide more energy than plant foods per unit of weight, this fact need not contradict what Nestle $(1999,215)$ has claimed, namely that, up to when our ancestors started farming about 10,000 years ago, there is 'substantial support for the predominance of plant foods in hunter-gatherer groups living in areas where plants could grow. Whereas no milk other than human milk may have been consumed before farming was introduced, there is sufficient evidence to support the view that hunter-gatherer societies consumed a greater proportion of animal foods than subsistence farming communities later did (Leonard 2014): without modern technology,

How to cite this book chapter:

Deckers, J 2016 Animal (De)liberation: Should the Consumption of Animal Products Be Banned? Pp. 167-190. London: Ubiquity Press. DOI: http://dx.doi.org/10.5334/ bay.g. License: CC-BY 4.0 
it was difficult for most sedentary populations to adopt dietary patterns that contained large quantities of animal foods.

Modern science and technology have allowed many populations to become more sedentary, to escalate the production of plant foods (through mechanical and chemical agriculture), to use newly acquired genetic knowledge to create modified feed crops and animal breeds in order to increase the quantity of animal products, and to develop intensive production systems of animal products (also known as factory farms or confined animal feeding operations-'CAFOs') as well as refrigeration and modern methods of transportation. Consequently, current diets of Western people in particular tend to include large quantities of animal products. These tend to be higher in total and saturated fats, as well as lower in mono-unsaturated and n-3 fats, than the animal products consumed by hunter-gatherer communities (Leonard 2014).

What we are currently witnessing is the globalisation of this typical Western diet through the influence of multinational corporations and of other market forces such as the acquisition of new capital by many populations, for example by many people living in China: until recently, many Chinese people could not afford to eat many animal products on a regular basis, and Chinese people were also much less exposed to the economic and political influences of large agricultural corporations that promote the consumption of such products. The recent increase in the consumption of these products in China was also facilitated by political shifts to a particular version of communism, followed by the rise of capitalist ideology, both of which undermined Buddhist questioning of such consumption. China's neighbouring country, India, has a long vegetarian tradition rooted in Hinduism and Buddhism, which emphasises the principle of ahimsa (non-violence) and a reverence for cows, in spite of the fact that some milk products have been consumed for a long time-incidentally, not without controversy, as the consumption of milk products was opposed by the Buddha's cousin, Devadatta (6th century BCE), and by those who followed his teachings (Simoons 1994, 6, 8). Like China, however, India is now moving rapidly away from its largely plant-based dietary tradition (Kasturirangan et al. 2014).

Even if their number is rising, it is nevertheless still the case that very few Western people adopt a vegan diet, and the number of people elsewhere who adopt dietary patterns that are totally or largely vegan is diminishing rapidly. There is no doubt that the moral case against veganism would be strengthened if it could be shown that vegan diets are unhealthy. Similarly, one might expect that the moral case in favour of such diets would be stronger if it could be shown that such diets are healthier than alternative diets. This is why I shall explore the healthiness of vegan diets in this appendix. Unlike in the main parts of this book, the concept of health is understood here in a narrow sense: the pivotal question that will be addressed is whether vegan diets are nutritionally adequate for those who adopt such diets, irrespective of their healthiness for others.

Before I embark on this task, it must be pointed out that any research into the nutritional value of vegan diets is hampered by several problems. One problem 
is the fact that many people who adopt these diets live in countries (for example India) where little attention has been paid to nutritional research, and few financial resources allocated to its funding. Another is that many people have traditionally adopted vegan diets out of necessity rather than out of choice. Up until recently for most, and even today for some, people ate what they ate because they lacked access to a diverse range of foods and, in many situations, found it harder to obtain animal products than to obtain other food. If many studied vegan populations adopt very restrictive diets because of pressing personal, social, or ecological constraints, it will be easy to find examples of deficient vegan diets, but much harder to find convincing evidence of the nutritional adequacy of such diets. The adoption of a very restrictive vegan diet may also be a symptom of a food disorder, for example anorexia. A further problem is the existence of a cultural bias against vegan diets (Sabaté 2003, 503S): as a result of this bias, dominant factions of societies that possess financial resources to study nutrition resist funding research that might undermine the status quo.

In spite of these obstacles, some research into the nutritional risks and benefits associated with vegan diets has taken place; I shall first engage with the question whether vegan diets could be healthy, and then move on to discussing the question whether well-planned vegan diets might actually be healthier than other diets.

\section{Might vegan diets be healthy?}

Many nutritionists claim that vegan diets can be healthy; the American Dietetic Association (ADA), for example, has argued that 'appropriately planned ... vegan diets ... are appropriate for individuals during all stages of the life cycle' (ADA 2009, 1266). To address this question in detail, however, it is necessary to focus on those dietary components that have frequently been suspected to be deficient in vegan diets. The components that deserve special scrutiny are: protein, calcium, vitamin B12, vitamin D, essential fatty acids, zinc, iodine, and iron.

\section{Protein}

Peas, lentils, and beans are good sources of protein that are readily available and relatively easy to grow in many parts of the world. It is important that vegans consume protein foods that contain the full range of essential amino acids overall; although there is no need for the full range of essential amino acids to be part of every meal (ADA 2009, 1268; McEvoy and Woodside 2010, 87), it is clear that we do need all essential amino acids to be healthy, which is why diets that rely on a very limited range of protein sources must be avoided. Although concern has been expressed over some populations that rely heavily on staples with limited quantities of protein, such as taro, cassava, and yams, 
Millward $(1999,259)$ has argued that 'cereal-based diets, especially those based on wheat and maize, supply protein levels considerably above the requirement level'. However, there is no evidence to suggest that those who consume relatively small quantities of cereals are likely to have deficiencies, provided that they consume other foods that contain significant quantities of protein. Overall, there is no evidence to suggest that vegans who eat a good range of plant foods are likely to lack in protein (Messina et al. 2004).

\section{Calcium}

Fruits and vegetables that contain relatively large amounts of potassium and magnesium decrease bone calcium resorption, whereas diets that include relatively large amounts of nuts and grains increase such resorption by producing a high renal acid load, mainly caused by residues of sulfates and phosphates (ADA 2009, 1269). Green leafy vegetables that are low in oxalate, including broccoli, kale, spring greens, and cabbage, tend to be high in calcium, as well as in vitamin $\mathrm{K}$, another important contributor to bone health (Messina and Mangels 2001, 663). The study of the Oxford-cohort of the European Prospective Investigation into Cancer and Nutrition (the 'Oxford-EPIC cohort') found that adult vegans who consume more than $525 \mathrm{mg}$ of calcium per day do not show higher fracture rates than omnivores (P. Appleby et al. 2007). There is no evidence that well-planned vegan diets fail to provide sufficient calcium, but there is evidence that diets that include adequate amounts of calcium and vitamin D are protective of bone health (Tang et al. 2007).

\section{Vitamin B12}

No plant foods are known to produce vitamin B12, or cobalamin, but those who eat plants inadvertently eat B12 as this vitamin is produced by micro-organisms (particularly Pseudomonas denitrificans and Propionibacterium shermanii) who live in symbiosis with many plants. The presence of vitamin B12 is essential for cell growth, and crucial for a healthy nervous system. Vitamin B12 deficiency leads to elevated plasma homocysteine (Hcy) concentrations (hyperhomocysteinaemia), a risk factor for neurological disorders and cardio-vascular problems, including pernicious anaemia and haematological disease (megaloblastic anaemia with demyelination of the central nervous system) (McEvoy and Woodside 2010, 90; Waldmann et al. 2005). Whereas our intestinal bacteria can synthesise B12, it is generally assumed that we should also consume products containing B12 (Li 2011).

Some studies have found that some vegans had inadequate intakes of B12, where particular concerns have been raised over the B12 status of older people due to their limited absorption capacity and of pregnant women due to 
their higher demands (Majchrzak et al. 2006; Waldmann et al. 2005; Donaldson 2000; ADA 2009; Piccoli et al. 2015). This is not a reason to eat flesh, as B12 binds with the protein in animal foods, impeding absorption, which is precisely why older people are better off with vegan sources of B12 (Norris and Messina $2011,31)$. Since the haematological symptoms of vitamin B12 deficiency may go undetected for a long time due to a high consumption of foods containing folate (folic acid), of which many vegans consume rather a lot through the consumption of things like oranges, green leafy vegetables, and beans, vegans must be very careful to ensure that their consumption of B12 is sufficient (ADA 2009, 1269). Many products, including cereals and yeast extracts, now exist that have been fortified with B12 produced through industrial fermentation of bacteria. In his assessment of the evidence, Sanders $(1999,267)$ has written that, provided that 'these foods are consumed regularly, the hazard of vitamin B12 deficiency is easily avoided'.

Norris and Messina $(2011,32)$ usefully point out that the human body only absorbs a tiny amount of B12 every time the vitamin is consumed, which is why they recommend the adoption of any one of these strategies for optimal consumption: 1/ two daily servings of fortified foods, providing 1.5 to 2.5 micrograms each; 2/ one daily supplement of at least 25 micrograms; 3 / one supplement of 1,000 micrograms twice weekly.

\section{Vitamin D}

Inadequate levels of vitamin $\mathrm{D}$ have long been known to contribute to bone problems such as rickets, but more recently have also been found to contribute to a range of other conditions, including fibromyalgia, rheumatoid arthritis, multiple sclerosis, depression, cancer, hypertension, and diabetes (Norris and Messina 2011, 47). Adequate exposure to sunlight can provide the body with all the vitamin $\mathrm{D}$ it needs, but overexposure must be avoided as ultraviolet irradiation is a significant contributor to skin cancer. Those people who are not regularly exposed to sunlight, as well as those whose bodies are limited in the uptake of vitamin $\mathrm{D}$, such as older and dark skinned people, must therefore consume products that have been fortified with vitamin $\mathrm{D}$ or take supplements (Craig 2009, 1629S; Stacey et al. 2005, 1444; Holick 2007). Vitamin D3 (cholecalciferol), used as a supplement, is usually derived from lanolin (sheep's wool) or fish oil, and is also found in some lichen and extracted from them by some companies, but the consumption of vitamin D2 (ergocalciferol)-produced from the ultraviolet irradiation of ergosterol from yeast-has been shown to be as effective in providing the human body with vitamin D (Holick et al. 2008).

Plasma 25-hydroxyvitamin D concentrations were measured in 2,107 participants of the Oxford-EPIC cohort, showing that vegans had lower concentrations of vitamin D, particularly during the winter months (Crowe et al. 2010). Whereas most participants in this study had concentrations that were deemed 
to be adequate, it is nevertheless very important to recognise that many people who live far away from the equator and who do not expose themselves frequently to sunlight (because of spending much time indoors and clothing) fail to meet recommended levels. This may be why Craig $(2009,1630 S)$ has expressed the view that a daily supplement of 5-10 micrograms of vitamin D would be 'highly desirable for elderly vegans'; however, some recent studies suggest that a higher dosage may be required to maintain optimal blood levels, which is why Norris and Messina $(2011,47)$ recommend 25 micrograms or 1,000 International Units (IUs) daily for people who do not benefit from adequate sun exposure.

\section{Essential fatty acids}

Omega-3 (or n-3) and omega-6 (or n-6) fatty acids are widely regarded to be beneficial for human health. The two most important ones of these are two short-chain polyunsaturated fatty acids: $\alpha$-linolenic acid (ALA), which the body can use to create other fats within the n-3 fatty acid family, and linoleic acid (LA), which the body can use to create other fats within the n- 6 fatty acid family. These two fatty acids are called 'essential' because they cannot be synthesised by the human body, but are required for healthy functioning. They must therefore be supplied by our diets. Enzymes in our bodies convert these short-chain fatty acids to long-chain n-3 and n-6 polyunsaturated fatty acids. ALA is converted (incidentally, not only by humans, but also by many other animals, including fish) to eicosapentaenoic acid (EPA), docosahexaenoic acid (DHA) and docosapentaenoic acid, with stearidonic acid (SDA) as an intermediate in the pathway; LA is converted to arachidonic acid (Saunders et al. 2012a).

The palaeolithic diets that were adopted by hunter-gatherers are estimated to have had an n-6:n-3 ratio of 1:1 to 2:1. Many people who live today, by contrast, overconsume LA (C. Williams and Burge 2006). The n-6:n-3 ratio of typical Western diets has been estimated to be around 15:1 to 17:1 (O’Neill 2010, 200). This is a serious problem, as overconsumption of LA impairs ALA conversion. Many people also underconsume ALA, which may cause deficiencies in the particularly important EPA and DHA (B. Davis and Kris-Etherton 2003). High intakes of trans-fatty acids, alcohol, and caffeine, as well as imbalanced diets and illness in general, may produce the same deficiencies in EPA and DHA. Such deficiencies are believed to cause cardio-vascular disease and cancer, as well as exacerbated pain associated with a range of conditions (Simopoulos 2002; von Schacky 2009; Christophersen and Haug 2011). They may also cause cognitive decline, age-related macular degeneration, and depression (Saunders et al. 2012a, 24S).

A clear message emerges from this. Vegans must make sure to consume adequate amounts of ALA, and avoid high consumption of products that inhibit the conversion of ALA, including products that contain relatively large quantities of LA. Accordingly, a recent study recommends that at least one unit of 
n-3 be consumed for every four units of n-6 (Saunders et al. 2012a, 24S). The authors of the study also recommend an ALA intake of $2.6 \mathrm{~g} /$ day for men and $1.6 \mathrm{~g} /$ day for women, whilst recommending the following daily intakes for infants and children: $0.5 \mathrm{~g}$ at $0-6$ months; $0.5 \mathrm{~g}$ at $7-12$ months; $1 \mathrm{~g}$ for children aged $1-3 ; 1.6 \mathrm{~g}$ for children aged $4-8 ; 2 \mathrm{~g}$ for boys aged $9-13 ; 2.4 \mathrm{~g}$ for boys aged 14-18; and $1.6 \mathrm{~g}$ for girls aged 9-18 (Saunders et al. 2012a, 24S). The main reason for the gender differences relates to the fact that males tend to convert ALA less efficiently (Childs et al. 2008).

Plant foods that are high in omega-3 fatty acids include chia, flax, canola (rapeseed), hemp, walnuts, perilla, and olive oil (Saunders et al. 2012a; O’Neill 2010, 201). Blackcurrant seed oil, derived from the seeds of Ribes nigrum, is rich not only in omega-3 fatty acids, but also in SDA, and the same applies to oil derived from plants belonging to the Echium genus, a collection of species within the Boraginaceae family (Li 2011). Genetically engineered soybeans that contain SDA have also been recommended (Saunders et al. 2012a), but their inclusion within a diet would depend on their acceptability, a debate that I touched upon briefly in section 2.11 and that I shall not engage with any further here. To ensure adequate consumption of ALA, Norris and Messina (2011, 89) recommend that adults consume three to four daily servings from this list: ' 1 teaspoon canola oil, 1/4 teaspoon flaxseed oil, 2/3 teaspoon hempseed oil, 1 teaspoon walnut oil, 2 teaspoons ground English walnuts or 2 walnut halves, 1 teaspoon ground flaxseeds, $1 / 2$ cup cooked soybeans, 1 cup firm tofu, 1 cup tempeh, 2 tablespoons soynuts'.

People with increased needs (for example pregnant and lactating women) and people with compromised conversion rates (for example people with diabetes or hypertension, and older people) may also benefit from consuming limited amounts of DHA- and-where available-EPA-fortified foods and DHA-supplements derived from microalgae (which can retro-convert to EPA inside the human body), as well as from consuming brown algae (kelp) oils (Saunders et al. 2012a; ADA 2009, 1268, 1271; Craig 2009, 1629S; Geppert et al. 2005). Norris and Messina $(2011,58,55)$ write that vegans over the age of 60 'should consider' a daily DHA (or a combination of DHA and EPA) supplement of 200 to 300 milligrams, a supplement dose that they are also 'inclined to recommend' at a frequency of every two to three days for those who are younger.

Although it may be unlikely to happen, overconsumption of DHA-rich products must be avoided, as this may raise total and low density lipoprotein (LDL) cholesterol, cause prolonged bleeding, and reduce immunity (Craig 2009, 1629S; Geppert et al. 2005; Sanders et al. 2006).

\section{$\operatorname{Zinc}(\mathrm{Zn})$}

Provided that it is present in the soil, many plant foods contain zinc. Plants that tend to be high in zinc are cereals and legumes. Unrefined whole grains provide 
higher concentrations than refined grains, as zinc can be found particularly within the outer layer of grains (Saunders et al. 2012b, 17S). Various ways to increase zinc uptake have been described, including soaking and sprouting beans, seeds, and grains, as well as leavening bread and consuming foods that contain citric acids (Lönnerdal 2000). Zinc absorption can be reduced by phytates (phytic acids), protein, and insoluble fibre, as well as by some minerals, including iron, calcium, and potassium (Li 2011). Whereas whole grains are higher in phytates than refined grains, the relative greater effect of phytates in the former is more than compensated for by the fact that whole grains are higher in zinc (Messina and Mangels 2001, 664). A study that compared 25 vegans with 20 omnivores found that the inhibitory effect of phytate failed to compromise zinc status as the bodies of people who take in little zinc appear to be able to increase zinc absorption and retention (Haddad et al. 1999).

As an aside, whereas it is good to be mindful that potassium may inhibit the absorption of zinc, it is nevertheless important to secure a sufficient intake of potassium as well. The following are listed as good sources of potassium by Norris and Messina $(2011,76)$ : beet greens, spinach, Swiss chard, cooked tomatoes and tomato juice, bananas, sea vegetables, orange juice, and legumes.

\section{Iodine}

Iodine deficiency affects more than two billion people. It is the leading cause of preventable mental retardation worldwide. Foetuses and breastfed children are particularly vulnerable as they depend on maternal iodine intake for thyroid hormone synthesis, which is essential for human neurological development. Thyroid iodine uptake is inhibited by perchlorate-an ubiquitous environmental contaminant-cigarette smoke, cruciferous vegetables (of the family Brassicaceae), and seaweeds of the genus Laminaria (including kombu) (Leung et al. 2011, e1304; Lightowler 2009, 433-434); there is also concern over the inhibitory effects of particular isoflavones found in soya and flaxseed. Both the underconsumption and the overconsumption of iodine can cause goitre (an enlargement of the thyroid gland) and hypothyroidism, but the latter can also cause hyperthyroidism (Norris and Messina 2011, 70-71). A small American study found, however, that in spite of the fact that a cohort of Boston-area vegans had relatively low urinary iodine levels, these low levels were not associated with thyroid dysfunction (Leung et al. 2011).

Provided that they have access to adequate nutrition, vegans should not suffer from iodine deficiencies. Iodine can be provided through plants grown on iodine-rich soil, the consumption of seaweed, and the consumption of iodised salt. As levels of iodine in seaweed vary considerably and are therefore unreliable, and as the overconsumption of salt must be avoided, Norris and Messina $(2011,72,89)$ recommend the use of supplements as their favourite strategy, where their recommendation for adults is that they take supplements of 75 to 
150 micrograms three to four days per week in order to meet a recommended daily allowance of 150 micrograms, whereas lower levels of 90 micrograms daily are recommended for very small children and higher levels of up to 290 micrograms daily for lactating women. They also recommend one quarter of a teaspoon of iodised salt per day as an alternative to supplementation. The development of a global strategy to ensure routine, adequate iodisation of foods which are commonly used that guards at the same time against excess intake of iodine, which negatively affects the thyroid gland (Lightowler 2009, 431), would seem to be appropriate in view of the scale of the problem of iodine deficiency. Some localities have already developed guidelines; in the USA, for example, vegan pregnant and lactating women have been recommended to supplement their diets with 150 micrograms of iodine daily (Leung et al. 2011, e1303).

\section{Iron}

Foods contain iron in two forms: haem iron and non-haem iron. Vegan foods only contain the latter, which is less easily absorbed by the body. Whereas iron deficiency can be a problem for vegans, it is more likely to be a problem for omnivores who consume large quantities of milk than for diet-conscious vegans. Good vegan sources of iron are dried fruit, sea vegetables, leafy green vegetables, and beans (Norris and Messina 2011, 64, 70). Vegans who consume a good range of fruit and vegetables in addition to foods that contain relatively large amounts of iron are unlikely to be affected by a deficiency as many fruits and vegetables contain large quantities of vitamin $\mathrm{C}$, as well as other organic acids, which enhances iron absorption. Retinol, carotenes, and alcohol have also been reported to increase iron absorption, whereas inhibitors include oxalates, phytates, and calcium, as well as the polyphenolics that are present in tea, some herbal 'teas', coffee, and cocoa (Ma et al. 2005; Siener et al. 2006; Hallberg and Rossander 1982; Li 2011; McEvoy and Woodside 2010, 88; ADA 2009, 1268). It is for this reason that Norris and Messina $(2011,70)$ recommend that people who drink tea and coffee only do so between meals rather than with their meals. As low iron status is moderately common in premenopausal women, these women need to make sure that their diets include good sources of iron, together with vitamin $\mathrm{C}$ to aid absorption (Key et al. 2006, 37). At the same time, there is evidence of the human body's ability to adapt to low iron intake by increasing absorption and decreasing losses (Hunt and Roughead 1999; Hunt and Roughead 2000).

\section{Taking stock}

The account presented above shows that vegan diets can fulfil all the nutritional requirements that are needed to support good health. Nutrients that present particular concerns are vitamin B12 and omega-3 fatty acids as few vegan foods 
that are currently used for human consumption contain these. Accordingly, vegans must make sure that they consume adequate portions of such foods. A nutrient that I have not mentioned, but that may be a concern, is selenium (Norris and Messina 2011, 76): as the selenium content of soil varies across the world, vegans must ensure that they do not restrict their diets to foods that are grown on soils that have low selenium levels. All in all, vegan diets can be adequate for all human beings, including children. Although small children with reduced stomach capacities may need to eat regularly and must ensure that they eat foods that are sufficiently high in energy density to provide sufficient calories, that are relatively easy to digest (for example by including cooked rather than raw foods), and that are not excessive in fibre (Messina and Mangels 2001, 662), many nutritionists adopt the view that vegan diets can be adequate for all human beings (Messina and Mangels 2001; Norris and Messina 2011; Van Winckel et al. 2011; ADA 1997).

\section{Might vegan diets be healthier than other diets?}

The claim has also been made that well-planned vegan diets may be healthier than other diets (Norris and Messina 2011, xv; B. Davis and Melina 2014, 29). It is this claim that I shall explore in the remainder of this appendix. One way in which this claim could be examined is by focusing on mortality differences between vegans and others. The problem, however, is that no studies exist of populations where omnivores share similar genetic profiles, similar lifestyle patterns, and similar social and environmental factors with a significant number of vegans. Nevertheless, a meta-analysis of seven prospective cohort studies - that is, studies which compare, usually over a long time, those who remain healthy with those who become ill-from the UK, Germany, California, the USA, the Netherlands, and Japan, including 124,706 participants, compared vegetarians with omnivores and found that all-cause mortality was $9 \%$ lower amongst vegetarians (T. Huang et al. 2012).

Whereas the fact that vegetarians benefit from increased longevity does not imply that this would also be the case for vegans, there is evidence that people who consume large quantities of fruits and vegetables-foods that tend to be more prominent in vegan diets-live longer than those who do not do so. Some evidence for this is provided by a Finnish study of 2,641 men who were aged between 42 and 60 and whose diets were assessed by four-day food intake records between 1984 and 1989. With a mean follow-up time of nearly 13 years, the study found that, after adjustment for major risk factors for cardio-vascular disease, those within the highest fifth for intake of fruits (including berries) and vegetables had a relative risk for all-cause death that was $34 \%$ lower than that of those in the lowest fifth (Rissanen et al. 2003). Several other studies found a positive association between diets that are relatively high in the consumption of fruits and vegetables, such as the traditional Mediterranean diet of people 
who lived in Pioppi (Italy) up to about four decades ago, and a reduction in mortality (Keys 1995; Benzie and Wachtel-Galor 2010). As diets that include a large proportion of fruits and vegetables have been shown to be healthier than diets that include relatively few of these foods, it has been estimated that a large number of premature deaths could be prevented amongst populations that consume large quantities of animal products by increasing the consumption of plant foods (Scarborough et al. 2012a).

In the remainder of this appendix I shall focus on studies that provide evidence for a difference between vegan and other diets in relation to the morbidity factors of obesity, bone health, cardio-vascular disease, diabetes, cancer, diverticular disease, Parkinson's disease, and insulin-like growth factor 1 (IGF1) and mTORC1 related diseases.

\section{Obesity}

Several studies have shown that vegan diets are associated with a reduced incidence of obesity as they tend to include fewer trans-fats (which are found mainly in processed foods with partially hydrogenated fats), fewer saturated fats (which can also be found in fully hydrogenated vegetable oils), and more dietary fibre (Rizzo et al. 2013; ADA 2009, 1274; McEvoy and Woodside 2010, 84; Spencer et al. 2003; Davey et al. 2003; Haddad et al. 1999). Obesity is a known risk factor for a wide range of health conditions, including cardio-vascular disease, type 2 diabetes, some cancers, and dyslipidaemia (WCRF/AICR 2007, 374-376). In addition, HIV patients may avoid or reduce lipodystrophy problems by adopting vegan diets (McCarty 2003b). In recent years, many companies in the dairy industry have responded to the challenges associated with rising rates of obesity by producing and promoting low fat alternatives. In spite of the reduction in fat, these products still contain large amounts of calories that are turned into fatty tissues if they are surplus to human energy requirements, thus contributing to increases in weight (Lanou 2009).

\section{Bone health}

The Oxford-EPIC study found that UK vegans had a $30 \%$ increase in fractures compared to other dietary groups in the UK and that $45 \%$ of the vegan group consumed less than $525 \mathrm{mg}$ of calcium per day, compared to only $6 \%$ in the other dietary groups (P. Appleby et al. 2007). When vegans whose consumption averaged more than $525 \mathrm{mg}$ of calcium per day were compared with other groups, however, fracture rates in this specific vegan group were about the same as those in the other groups.

Cows milk is frequently recommended for bone health. However, in a study of 72,337 postmenopausal women that followed up participants for hip 
fractures for 18 years, it was found that neither a high calcium diet nor cows' milk consumption was associated with a reduced risk of hip fracture (Feskanich et al. 2003). An earlier, retrospective study found that hip fractures are higher in countries with high protein consumption from animal products (Abelow et al. 1992).

This finding tempted Lanou $(2009,1639$ S) to speculate that high consumption of animal products may undermine bone health. For three reasons, it is hard to conclude this from the Abelow et al. (1992) study. Firstly, the study estimated protein consumption for whole populations, rather than for the study groups; estimated intakes of animal protein may therefore differ greatly from what those who suffered hip fractures actually consumed. Secondly, the interpretation ignores that many countries where relatively large quantities of animal products are consumed tend to have high life expectancies (Kannus et al. 1996); the fact that rates of hip fractures are higher in countries where lots of animal products are consumed may therefore simply be explained by the fact that life expectancies are higher within those countries. Thirdly, the possibility that cultures that rely heavily on animal products may have different lifestyle factors that contribute to fracture risks should not be ignored (Calvez et al. 2012).

In spite of these reservations, limited evidence in support of Lanou (2009)'s hypothesis comes from a more recent, prospective study, which is interesting as it makes a direct comparison between fracture rates and bone mineral density loss in vegans and omnivores. The study, which took place in Ho Chi Minh City (formerly Saigon), compared the rate of femoral neck bone mineral density loss and morphometric vertebral fractures of 88 vegan and 91 omnivorous women over the age of 50 two years after baseline measurement. Groups were matched at baseline, but the vegans had significantly lower dietary intakes of calcium and vitamin $\mathrm{D}$, as well as of total protein and fats. In spite of their lower consumption of calcium and vitamin $\mathrm{D}$, this study found that there was no difference in fracture rates between vegans and omnivores, but that 'higher intakes of animal protein and lipid' (fat) were associated with greater bone loss (Ho-Pham et al. 2012, 75), a finding that the authors relate to earlier research that attributes a causal role in bone loss to the presence of high levels of acid in animal protein (Barzel and Massey 1998). Given the small number of participants that were involved and the specific genetic, cultural, and environmental context, it is not possible, however, to conclude that vegans are more likely to have healthier bones that are less prone to fractures than omnivores.

Further research has also revealed that high consumption of protein may be a risk factor for fractures not per se, but only when it is combined with low consumption of calcium (Burckhardt 2013). On the other hand, through increased consumption of fruits and vegetables, vegans tend to have a lower renal acid load, which reduces urinary calcium excretion and bone resorption (New 2003). In this respect, high consumption of vegetables and fruits with high potassium, magnesium, and vitamin $\mathrm{K}$ contents may be particularly desirable 
(Calvez et al. 2012; Tucker et al. 2001; Booth et al. 2000; Feskanich et al. 1999). A further reason why vegans may be protected relates to the fact that vegans do not consume preformed vitamin $\mathrm{A}$, which is known to cause a reduction in bone mineral density if it is consumed in large amounts (Burckhardt 2015).

Whereas bone health is not necessarily undermined by low calcium intakes, it must be emphasised that calcium is the main mineral in human bones. Adequate consumption of calcium is one factor that contributes to good bone health, even if it does not guarantee it as the rate at which calcium is absorbed is determined largely by other dietary factors. One of these factors is vitamin D status, the importance of which was highlighted earlier. Another is adequate protein consumption. The importance of the latter is borne out by a study of 1,865 women from Canada and the USA who were followed up over 25 years, where, of the $40 \%$ who adopted a vegetarian diet, those with the highest protein consumption had the lowest risk of wrist fractures (Thorpe et al. 2008).

\section{Cardio-vascular disease}

Cardio-vascular diseases are the most common causes of mortality. Most cardio-vascular diseases result from venous or arterial blockages (thrombosis). These occur by a rupture of atherosclerotic plaque and result in tissue damage from blood starvation. Cerebrovascular and ischaemic heart diseases are the two most common types of cardio-vascular disease.

A meta-analysis that included 124,706 participants recruited for seven prospective cohort studies that compared vegetarians with omnivores in the UK, Germany, California, the USA, the Netherlands, and Japan found that vegetarians had a $29 \%$ lower mortality risk for ischaemic heart disease (T. Huang et al. 2012). This is in line with findings from a meta-analysis of five prospective studies that compared data for 76,172 people from Germany, the UK, and the USA, which found that the mortality rate from ischaemic heart disease was $24 \%$ lower in vegetarians than in non-vegetarians after a mean follow-up of just over ten years and a half (Key et al. 1999). Although the death rate for ischaemic heart disease was slightly higher for the vegans than for the vegetarians in this latter meta-analysis, the risk ratio for death from cerebrovascular disease for vegans was only about half that for those who ate animals' flesh at least once a week.

Two large, and ongoing, cohort studies in particular have been widely reported with regard to diet-associated cardio-vascular disease risk. The first is the Oxford-EPIC study; the second a study ('the AHS-2 study') from the USA and Canada with a cohort of 73,308 Seventh-day Adventists who were recruited at churches between 2002 and 2007 and followed up over more than five years (Orlich et al. 2013).

The Oxford-EPIC study has documented that self-reported hypertension was lowest amongst vegans, whilst a study of blood pressure in a sub-cohort of 
8,663 participants who reported not to suffer from hypertension found that the 612 vegans in that sub-cohort showed lower systolic and diastolic blood pressures than people in any other dietary category in that sub-cohort, which could only partly be attributed to differences in body mass, i.e. the fact that the vegans tended to be leaner (P. Appleby et al. 2002). In 2013, the authors of the study reported that a vegetarian group (which included vegans), which comprised $34 \%$ of a total sub-cohort of 44,561 people living in England and Scotland, had a $32 \%$ lower risk of ischaemic heart disease after a follow-up of just over 11 years than the omnivores in the same sub-cohort when adjustment for all confounding factors apart from body mass index (BMI) was performed, and a 28\% lower risk when BMI was factored in (Crowe et al. 2013).

Similar findings are reported in the AHS-2 study (Orlich et al. 2013). Compared to the group of omnivores, deaths from ischaemic heart disease and cardio-vascular disease were, respectively, $10 \%$ and $9 \%$ lower amongst the group of 3,533 'vegan'-defined here as those who reported to consume animal products less than once a month-women, whereas the group of 2,015 'vegan' men experienced risk reductions of, respectively, 55\% and $42 \%$. For a sub-group of this cohort, comprising 500 white subjects, it was found that the group of 49 'vegans' had a $63 \%$ lower risk of suffering from hypertension (where someone suffering from hypertension was defined as someone who either took medication for it or someone who had a systolic blood pressure exceeding 139 $\mathrm{mmHg}$ or a diastolic blood pressure exceeding $89 \mathrm{mmHg}$ ), which was only partly accounted for by differences in body mass (where 'vegans' tended to be leaner) (Pettersen et al. 2012).

Whereas the Oxford-EPIC and the AHS-2 studies concern Western populations, similar results were obtained in a Chinese study, where healthy men who consumed no animal products other than milk were found to have lower risks of cardio-vascular disease than omnivorous men (Yang et al. 2012).

Why is it that vegans may be less prone to cardio-vascular disease than omnivores? Both obesity and hypertension may play a role in this difference, as both high BMI and high blood pressure have been associated with elevated risk. Another reason relates to levels of cholesterol. Low density lipoprotein (LDL) and high density lipoprotein (HDL) are the main cholesterol components that are found in our blood; a low level of the former and a high level of the latter are generally thought to benefit cardio-vascular health. LDL can oxidise, promoting plaque formation and hardening of the arteries, but this can be undermined by high levels of HDL. Vegan diets may protect against cardio-vascular disease because they do not contain animal products, which tend to be relatively high in substances that elevate LDL cholesterol, including total and saturated fat (Fung et al. 2010; Bernstein et al. 2010; Norouzy et al. 2011). Vegan diets are also generally higher in fibre, which has been found to reduce LDL cholesterol (Jenkins et al. 2001). In relation to this, research has found that the consumption of whole grains, which-unlike refined grains-include the bran, germ, and endosperm, and are relatively rich in fibre, reduces cardio-vascular 
risk factors (Liu et al. 1999; Park et al. 2011). Vegan diets also tend to be relatively low in bio-available phosphate, where high phosphate levels are associated with increased risk (McCarty 2003a). Vegans must be careful, however, to avoid overconsumption of refined carbohydrates (as for example white-flour products, white rice, and sugar), as this reduces HDL, which removes excess LDL cholesterol from the bloodstream (O'Neill 2010, 202-203). As mentioned before, they must also be careful to maintain adequate levels of vitamins B12 and $\mathrm{D}$, as well as a good balance of n-6 over n-3 fatty acids, as deficiencies in these domains have been associated with elevated risks of cardio-vascular disease (Li 2011; Woo et al. 2014; Bouillon and Verlinden 2014).

Vegans may benefit not only from lower LDL levels, but also from the fact that they tend to remove detrimental components ('atherogenic remnants') more quickly from the blood (Vinagre et al. 2013). In addition, several studies have associated reduced risks of cardio-vascular disease with high intakes of fruits, vegetables, and nuts (Finks et al. 2012; Takachi et al. 2008; He et al. 2006; Mozaffarian et al. 2011; Hu 2003; Jenkins et al. 1997; Sacks et al. 1999). This stems at least in part from the fact that diets that are high in nuts and in plant sterols are known to reduce total and LDL cholesterol levels (Katan et al. 2003; Mukuddem-Petersen et al. 2005; Sabaté et al. 2010).

\section{Diabetes}

Although some studies have linked the development of type 1 diabetes to the consumption of dairy products (Dahl-Jørgensen et al. 1991; Banwell et al. 2008), a meta-analysis of studies pointed out that no causal link has been established (Agostoni and Turck 2011). However, a more recent study suggests that consumption of cows' milk very early in life may trigger type 1 diabetes if it is accompanied by exposure to enterovirus infections in early life (Lempainen et al. 2012).

More evidence exists on the positive benefits of vegan diets for the prevention and treatment of type 2 diabetes, as well as of the associated cardio-vascular diseases (Kahleova and Pelikanova 2015; Tonstad et al. 2009; Marsh and Brand-Miller 2011; Salas-Salvadó et al. 2011). As weight is a major risk factor for the development of this condition, vegans are less likely to develop type 2 diabetes because of their lower weight (Fung et al. 2004; Trapp and Levin 2012). However, several studies show that there are other factors why vegan diets may prevent type 2 diabetes, such as the fact that no red and processed flesh is consumed, and that more whole grain foods and nuts may be consumed, all of which factors have been associated with reduced diabetes risk (Pan et al. 2011; Marsh 2011).

Vegan diets have also been shown to help in the treatment of type 2 diabetes by lowering total and LDL cholesterol and by controlling lipid levels, for example by reducing triglycerides, a type of fat that is also associated with a greater 
risk of heart disease (Jenkins et al. 2006; Barnard et al. 2006; Barnard et al. 2009, 1594S; Tonstad et al. 2009; Vinagre et al. 2013). Many vegan diets have a low glycaemic index (GI) and a fairly low glycaemic load. The GI is a measure of the effect of carbohydrate-containing foods on blood glucose response (i.e. how quickly the body converts carbohydrates into energy) after their consumption (Jenkins et al. 1981), and the glycaemic load is the product of the amount of foods consumed and their glycaemic index (Finks et al. 2012, e70). People who consume large quantities of foods that have a high GI are thought to be at increased risk not only of diabetes and cardio-vascular disease, but also of a number of conditions-sometimes grouped under the label of 'metabolic syndrome'-including obesity, hypertriglyceridemia, and low HDL cholesterol (Finley et al. 2010; Ludwig 2002; Finks et al. 2012). It has also been found that obesity reduces tolerance of diets with high glycaemic load (Liu et al. 2000). In relation to diabetes, diets with high GI values are associated with greater insulin resistance and a greater incidence of hypoglycaemia amongst those who are treated with insulin (Willett et al. 2002; Ebbeling et al. 2007).

In a randomised controlled trial of a duration of five months, whereby 99 people with diabetes were divided into a group of 49 who were asked to follow a vegan diet and a group of 50 who were asked to follow a diet recommended by the American Diabetes Association, the overall GI of the vegan group's diet was significantly lower than that of the other group's diet (Turner-McGrievy et al. 2011). The associated reduction in body weight, together with the reduced fat content (and the associated reduction in intramyocellular lipid-a contributor to insulin resistance) and increased fibre content of the vegan diet, was thought to result in better glycaemic control (Turner-McGrievy et al. 2011, 1472). The vegan group also managed to reduce their medication significantly more than those who belonged to the other group, a significant finding in light of the fact that some hypoglycaemic drugs contribute to weight gain (Barnard et al. 2006; Barnard et al. 2009). All this does not imply that one's dietary glycaemic index is necessarily lowered by the adoption of a vegan diet, as Norris and Messina $(2011,185)$ rightly point out that 'the key is to choose carbohydrate-rich foods with low GIs, which means eating more unprocessed, whole plant foods in place of refined carbohydrates'.

\section{Cancer}

It is highly probable that many vegan diets are less likely to cause cancer than other diets are. The Oxford Vegetarian Study and the Oxford-EPIC study provide evidence for this claim (Key et al. 2009a). The former study recruited 11,140 vegetarian and non-vegetarian participants throughout the United Kingdom between 1980 and 1984 . The latter study recruited a much larger number of participants between 1993 and 1999, and is part of a much larger, multicentre, prospective study with 519,978 subjects overall, carried out in 
23 centres from 10 European countries (Denmark, France, Germany, Greece, Italy, the Netherlands, Norway, Spain, Sweden, and the United Kingdom). Data from the Oxford Vegetarian Study and the Oxford-EPIC study were combined, resulting in a cohort of 61,566 people (15,571 men and 45,995 women) who were followed up to 2007; participants were separated into three dietary groups on the basis of their answers to four questions, collected by means of an intake questionnaire: 32,403 omnivores, 8,562 fish eaters (who did not eat any other animals' flesh), and 20,601 vegetarians (Key et al. 2009a).

Before looking at the evidence of this combined study, it must be recognised that this study is not free from methodological concerns. Since it is a longitudinal study, it is quite possible that dietary patterns varied significantly over the large number of years that participants were followed up. A second problem is that actual diets may differ from reported diets. From a personal lunch-time conversation with a participant in the EPIC study, I found out, for example, that he had chosen the vegetarian group, whereas he actually ate fish. A third problem is that the more subtle distinctions between the kinds of foods that people eat are ignored by the fact that the questionnaire only aimed to distinguish between three dietary categories, omitting a vegan diet category. A fourth problem is that participants appeared to be particularly health conscious whichever diet they adopted, as death rates were significantly lower (at 52\% of the general population's death rates in the Oxford-EPIC study) than that in the general British population (Key et al. 2009b). These problems impair the ability to generalise results from this study group to other people.

In spite of these difficulties, it is significant that the study found that the overall cancer incidence amongst vegetarians was about $12 \%$ lower than the incidence amongst omnivores (Key et al. 2009a), which is in line with the $18 \%$ reduction that was found in a recent meta-analysis of seven prospective cohort studies that compared vegetarians with omnivores in the UK, Germany, California, the USA, the Netherlands, and Japan (T. Huang et al. 2012). The combined Oxford study found lower incidences in the vegetarian group for ovarian and bladder cancers, as well as for cancers of the lymphatic and haematopoietic tissues and for stomach cancers (of which there were only 49 cases), but the risk of cervical cancer-of which there were only 50 cases-was more than twice as high in the vegetarian group than in the group of omnivores. The authors speculate that this higher observed incidence of cervical cancer might be related to non-dietary factors, for example differences between groups in attendance for cervical cancer screening. They did not find a significant difference between dietary groups in relation to the incidence of colorectal cancer, which contrasts with a study that aggregated EPIC data from 10 European countries, which found that high consumption of red and processed flesh was associated with a higher risk of colorectal cancer (Gonzalez and Riboli 2006, 229). Similarly, an expert systematic review in the USA deemed that the evidence of the increased risk for colorectal cancer associated with consuming red and processed flesh was convincing (WCRF/AICR 2007, 116, 382). The same review judged that 
there was limited evidence for a positive association between the consumption of red and/or processed flesh and increased risks of cancers of the oesophagus, stomach, pancreas, lung, endometrium, and prostate (WCRF/AICR 2007, 116). Another expert review adds breast, bladder, and oral cancer (Anand et al. 2008).

The link between the consumption of animal products and cancer has also been studied by Ganmaa and Sato (2005), who correlated the incidence rates for breast, ovarian, and corpus uteri cancers (using data detailing cancer incidence between 1993 and 1997) with food intake in 40 countries-even if food consumption was merely estimated by means of 1961-97 FAOSTAT data. They found a positive link between the consumption of animal products and these hormone-dependent cancers, a finding that is corroborated by other studies (Larsson et al. 2006). Ganmaa and Sato (2005) express particular concern with the consumption of milk from pregnant cows. As many cows in the dairy industry are almost continuously pregnant, their milk expresses high levels of oestrogen and progesterone (hormones which are known to stimulate the mammary gland), which are hypothesised to increase the risks associated with these cancers (Ganmaa and Sato 2005).

In many situations, men may not benefit from the consumption of dairy products either. A World Cancer Research Fund (WCRF) and American Institute for Cancer Research (AICR) joint expert review concluded that 'there is limited evidence suggesting that high consumption of milk and dairy products is a cause of prostate cancer', but also that cows' 'milk probably protects against colorectal cancer' (WCRF/AICR 2007, 129). This is more or less the opposite of what was found in a longitudinal study of 4,383 English and Scottish children who participated in a family food study between 1937 and 1939: no positive link between high cows' milk consumption and prostate cancer risk was found, but the study did find a near-tripling in the odds of colorectal cancer amongst those who had been raised in households with high dairy consumption (van der Pols et al. 2007). Some other studies, however, also found a positive link between high consumption of dairy and prostate cancer risk (N. Allen et al. 2008; Chan et al. 2005; Torfadottir et al. 2011). Much has been written on the latter issue, but little clarity has been provided because of the high likelihood of confounding factors. An analysis of pooled data from 45 observational studies, supported by a grant from National Dairy Council (Rosemont, Illinois), found no increased risk (Huncharek et al. 2008).

Apart from the fact that no dairy products are consumed, many other reasons have been provided in support of the view that vegan diets are cancer-protective. One is the fact that vegans are less likely to be obese (WCRF/ AICR 2007). Expert reviews also indicate that diets that are high in fruits and vegetables are associated with decreased cancer risk because of the higher levels of health-promoting substances (such as ascorbic acid, carotenoids, and flavonoids) and a lower level of some carcinogenic components that have been found in some animal products, such as dioxins (WCRF/AICR 2007; Craig 2009; Dewell et al. 2008; ADA 2009). 
A significant concern with many studies that explore relative cancer risks of different populations is that they fail to distinguish between vegetarians and vegans. Consequently, relatively little is known as yet about the benefits or disadvantages of vegan diets. The vegetarian group in the study that combined data from the Oxford Vegetarian Study and the EPIC-Oxford cohort, for example, included both vegetarians and vegans, resulting in a failure to identify the relative cancer risk of the latter (Key et al. 2009a). To alert the reader to this issue, the authors write that to explore the hypothesis that the consumption of dairy products may increase prostate cancer risk 'we would need to examine the cancer rates among vegans', but they are not consistent in their failure to separate vegans from vegetarians as they add that 'there are currently too few cancers [amongst vegans in their study] to be informative' (Key et al. 2009a, 195); what they may have meant to say is that there were too few vegans in the study to allow for generalisations to be made about vegan diets. As stated in the paper, however, the claim is informative. In spite of the fact that generalisations from studies of small populations are inappropriate, the fact that very few cancers were identified amongst vegans must be considered to be good news. In 2014, the Oxford team did report findings separately for the 2,246 vegans who were part of a sub-cohort of 61,647 British people who were followed up for almost 15 years (Key et al. 2014). During this time, there were 4,998 incidents of cancer, and the incidence was $19 \%$ lower in the vegan group than in the omnivorous group. Another study that has looked at vegans as a separate group is the AHS-2 study, which has reported a $16 \%$ reduction of risk amongst vegan Adventists compared to omnivorous Adventists (Orlich et al. 2013).

Overall, it is safe to conclude that many vegan diets are associated with a lower incidence of cancer than many other diets, even if the jury is still out on what the ideal diet might be to protect against cancer (Norris and Messina 2011, 176-178).

\section{Diverticular disease}

Diverticular disease includes two diseases of the colon (large intestine or large bowel): diverticulosis (the presence of pockets or pouches) and diverticulitis (infected or inflamed pockets or pouches). A study published in 1979 explored the incidence of diverticular disease in two groups of southern English people who did not experience any symptoms of the disease: 56 vegetarians were compared with 264 non-vegetarian volunteers. When radiographs of the participants' colons were analysed by a consultant radiologist who knew neither the participants nor their diets, $12 \%$ of the former group and 33\% of the latter group were diagnosed to suffer from diverticular disease (Gear et al. 1979). In the Oxford-EPIC cohort, a sub-cohort of 15,459 participants, combining vegetarians and vegans, was found to have a $30 \%$ reduced risk of diverticular disease compared with the sub-cohort of 31,574 omnivores (Crowe et al. 2011). When 
the vegan participants were isolated from the vegetarians, the researchers found a $72 \%$ lower risk for the former compared to the omnivores in the study. While these findings have primarily been associated with the fact that vegetarians and vegans tend to consume more fibre, different studies (with, arguably, participants less health-conscious than participants in the Oxford-EPIC studies) found that, after adjusting for differences in dietary fibre between study participants, high consumption of total fat or of red flesh (Aldoori et al. 1994), the consumption of flesh from sheep and cows as well as milk products (Manousos et al. 1985), and the 'long-term and frequent' consumption of flesh (Lin et al. 2000) were linked with diverticular disease. Whereas only the Aldoori et al. (1994) study was a prospective cohort study-the ones by Manousos et al. (1985) and by Lin et al. (2000) being small case-control studies-these findings lend strong support for the view that vegan diets that tend to be high in fibre are much less likely to cause diverticular disease than many omnivorous diets.

\section{Parkinson's disease}

On the basis of population-based studies, McCarty (2001b) found that Parkinson's disease was less prevalent in sub-Saharan Africa, rural China, and Japan. A similar observation was made by de Lau and Breteler (2006), who report that the incidence of Parkinson's is lower in East Asian populations, including Chinese, Taiwanese, and Japanese populations, than in Western populations. McCarty (2001b) also reported that the incidence of Parkinson's amongst African Americans was very similar to that of white Americans, suggesting that the low incidence of Parkinson's amongst sub-Saharan Africans may not stem from genetic factors. As sub-Saharan and East Asian populations consume relatively few animal products, McCarty (2001b) suggests that vegan diets may be protective and that they may even be therapeutically beneficial through a number of mechanisms, including the promotion of vascular health and blood-brain transport of L-dopa, as well as through caloric restriction, which was found to protect the central dopaminergic neurons of mice. A different study, funded by Syngenta Crop Protection, reviewed the epidemiological literature, as well as the literature on risks and protective factors, concluding that little is known as yet about the aetiology of Parkinson's disease, but that there is some evidence that the consumption of dairy products increases risk (Wirdefeldt et al. 2011). A very small Indian study, however, did not find a reduction in Parkinson's for those who adopted a vegetarian diet (Behari et al. 2001). In his review of the literature, Giovanni $(2009,326)$ comments that 'data regarding the prevalence of Parkinson's disease in vegetarian or vegan groups or relative clinical findings are not available as yet'. Accordingly, the view that a vegan diet might be protective of Parkinson's is no more than an interesting hypothesis at the present time. 


\section{Insulin-like growth factor 1 (IGF-1) and mTORC1 related diseases}

Insulin-like growth factor 1 (IGF-1) is a growth-stimulating hormone that is found in the human body. 'mTORC1' refers to mammalian target of rapamycin complex 1, a nutrient-sensitive enzyme that responds to a range of signals in the human body, including IGF-1.

Overproduction of IGF-1 has been associated with many diseases (Hoppe et al. 2006). IGF-1 is a key factor involved with episodes of rapid growth during childhood; the growth acceleration hypothesis claims that IGF-1 contributes to the development of a range of diseases that may not manifest themselves until much later in life (Singhal and Lucas 2004). Diets that increase IGF-1 levels in the blood have also been associated with some cancers, including colorectal and breast cancer (O’Neill 2010, 200).

Studies that compared vegan with other study participants have found that vegans had lower levels of IGF-1 (Fontana et al. 2006; N. Allen et al. 2002). Two cross-sectional analyses of the EPIC-study found that the production of IGF-1 was particularly stimulated by the consumption of dairy products (Norat et al. 2007; Crowe et al. 2009). The same conclusion was reached in a much larger study that combined findings from 15 cross-sectional studies and 8 randomised controlled trials (Qin et al. 2009). Dairy products have come under increased scrutiny not only because of their role in IGF-1 stimulation, but also because they, as well as animals' flesh, contain large quantities of calories and leucine. Together with products that have a high glycaemic load (including hyperglycaemic carbohydrates), products that are high in calories and leucine and that stimulate IGF-1 are thought to play a major, synergistic role in the activation of mTORC1 (Melnik 2012). This has been held to cause or worsen acne, a skin disease that prevails amongst more than $85 \%$ of teenagers in Western countries, and that is absent amongst people who eat palaeolithic diets, such as the inhabitants of Kitava, one of the Trobriand Islands of Papua New Guinea (Melnik 2012, 20-21; Lindeberg et al. 1999). Increased mTORC1-signalling has also been linked with a number of other Western health concerns, including obesity and type 2 diabetes (Shaw and Cantley 2006; Zoncu et al. 2011). Men who suffer from severe, long-lasting acne have also been found to have an increased risk of developing prostate cancer later in life (Sutcliffe et al. 2007). Laboratory experiments, including experiments with mice, have suggested that this may stem from the possibility that long-term hyperstimulation of mTORC1-signalling promotes the development of cancer tumours (Nardella et al. 2009; Wang et al. 2011).

Critical scrutiny of the Kitavans' diet around 1990 reveals that they ate a diet that contained mainly tubers that provide carbohydrates with a low glycaemic index (such as yam, sweet potato, taro, and manioc), as well as fruits, vegetables, coconuts, and fish (Lindeberg et al. 1999, 1216). In a randomised controlled trial with patients who suffered from ischaemic heart disease combined with either glucose intolerance or type 2 diabetes, such a diet has also been shown to improve glucose tolerance more than a Mediterranean-style diet that 
included whole grains and low-fat dairy products (Lindeberg et al. 2007). Compared to a Swedish control group, it was also found that Kitavans consumed a much smaller amount of mono-unsaturated fats and a higher amount of n-3 fatty acids (Lindeberg et al. 1999). In light of these studies and the connection between mTORC- 1 and a range of Western diseases that are rare or absent amongst Kitavans, the adoption of a vegan diet that is similar to the palaeolithic diet that was adopted by the Kitavans around 1990 has been recommended (Melnik 2012). One reason why such a diet is low in foods with a high glycaemic index is that it contains little fructose-which is present in many processed foods through the widespread use of high fructose corn syrup (Melnik 2012, 29; McCarty 2011; Seneff et al. 2011).

A vegan diet that is similar to the traditional Kitavan diet may also protect against a number of ageing-associated diseases, including Alzheimer's disease (Seneff et al. 2011; McCarty 2001a; McCarty 2003c). Alzheimer's disease patients have been shown to have elevated levels of IGF-1 (Melnik 2011). This may help to explain why, when 2,148 New Yorkers without a diagnosis of dementia who were at least 65 were followed up over a period of nearly four years, it was found that the incidence of Alzheimer's was greater amongst those who ate the largest quantity of animal products (Gu et al. 2010).

\section{Other benefits and concerns}

A further benefit for young children is that a vegan diet avoids the consumption of cows' milk, which not only is low in iron, but also causes occult intestinal blood loss in about $40 \%$ of children below the age of one, and which contains high quantities of calcium as well as casein and other proteins that all inhibit the absorption of dietary non-haem iron (Ziegler 2011, 38S-40S). Casein has also been found to inhibit the absorption of zinc (Lönnerdal 2000). These concerns may help to explain why nutritionists do not recommend the consumption of cows' milk for children below the age of one (Millward and Garnett 2010, 104). Middle ear infection (otitis media) has also been found to be more severe and more common amongst children with cows' milk allergies (Juntti et al. 1999). Such allergies are by no means restricted to children as many people are lactose intolerant, lacking sufficient quantities of the lactase enzyme within the lining of the small intestine to allow the body to absorb lactose, whilst some people are also allergic to other components in dairy products (Millward and Garnett 2010, 104-105). In light of their hypothesis that the continued production of lactase throughout adulthood may only have developed in northern Europeans about a thousand years ago, Norris and Messina $(2011,43)$ argue that the concept of 'lactose intolerance' stems from a Western bias as good lactose tolerance may be the exception, rather than the rule.

Limited evidence has been presented to support the view that vegan diets may also reduce the risk of cataracts, dementia, gallstones, kidney disease, 
and rheumatoid arthritis (B. Davis and Melina 2014, 72-80). As many toxic substances accumulate inside the bodies of animals, vegan diets also tend to have lower levels of many toxic substances, including biodegradation-resistant organic environmental pollutants, such as polychlorinated dibenzo-p-dioxins (PCDDs), polychlorinated dibenzofurans (PCDFs), and polychlorinated biphenyls (PCBs), as well as of toxic heavy metals, such as mercury (Schecter et al. 1997; O’Neill 2010, 201).

In spite of these benefits, recent research that included a sample of 422 vegans from the Oxford-EPIC cohort revealed that vegans had relatively high circulating concentrations of uric acid, which may contribute to the development of gout, chronic kidney disease, cardio-vascular disease, and cancer; these high concentrations of uric acid are attributed to the exclusion of dairy products and to low calcium consumption (J. Schmidt et al. 2013). The authors are cautious, however, about the possible existence of causal connections between uric acid and these diseases, and they add that concentrations can be lowered through increased calcium consumption.

An additional concern for people with small stomach capacities, such as small children, is that vegan diets can be bulky due to increased consumption of dietary fibre, which can cause early satiety. Accordingly, McEvoy and Woodside $(2010,86-87)$ advise that vegan children take frequent meals and snacks, and that foods that are high in fat, such as nuts and nut butters, be used to provide sufficient calories and protein. For those who suffer from nut allergies, however, adequate substitutes must be used.

\section{Conclusion}

After a brief introduction, I argued in the second part of this appendix that vegan diets can be nutritionally adequate, but that vegans must make sure to consume foods that contain adequate amounts of vitamin B12 and omega-3 fatty acids as the former cannot be obtained from plants and the latter are present in significant quantities only in a few common vegan foods. The former can be obtained by consuming products that contain the B12 vitamin. Adequate consumption of the latter is facilitated by the consumption of plants and plant foods that have relatively high levels of omega-3, such as chia, flax, canola (rapeseed), hemp, walnuts, perilla, olive oil, blackcurrant seed oil, and plants in the Echium genus, as well as by the consumption of brown algae (kelp) oils. People with specific dietary requirements, such as young and old people, must make sure that they eat sufficient foods that are relatively rich in calories and relatively easy to digest, such as cooked foods.

The question whether vegan diets might be healthier than other diets was addressed in the third part. The evidence to support the possibility that vegan diets might be healthier is limited. Factors that complicate the development of our understanding include the facts that relatively few people adopt vegan 
diets, that some people's adoption of vegan diets may be triggered by psychological illness, and that many are biased against vegan diets. In spite of these limitations, there is sufficient evidence to conclude that many diets that are high in fruits and vegetables are associated with many health benefits, including reductions in cardio-vascular disease and some types of cancer.

Whereas this appendix has discussed scientific evidence for and against vegan diets, it has not answered the question of what a good vegan diet is, at least not in detail. For those who seek more practical advice on what kind of vegan diet to adopt to meet nutritional requirements, I recommend the books Becoming Vegan (B. Davis and Melina 2014) and, particularly, Vegan for Life (Norris and Messina 2011). 